\title{
FREE-AMPLITUDE UPPER LIMB EXERCISES AFTER MASTECTOMY AND IMMEDIATE RECONSTRUCTION ARE BENEFICIAL AND SAFE: RANDOMIZED CLINICAL TRIAL
}

Samantha Karlla Lopes de Almeida Rizzi', Cinira Assad Simão Haddad¹, Simone Elias', Afonso Celso Pinto Nazário', Gil Facina1

'Universidade Federal de São Paulo - São Paulo (SP), Brazil.

Introduction: Physical therapy plays an important role in the prevention of motor and functional complications in the postoperative period of breast cancerl, reestablishing the motor function and improving the quality of life of patients2. However, there is no randomized study about the different physical therapeutic approaches in patients submitted to mastectomy and immediate alloplastic reconstruction. Objectives: To assess the impact of clearance for free-amplitude upper limb exercises, 15 or 30 days after surgery, on the movement amplitude (MA) of shoulder, pain and upper limb function; on the incidence of dehiscence, seroma, infection and necrosis; and on the need for reoperation in patients after mastectomy and immediate reconstruction with alloplastic material. Methodology: Sixty women who underwent mastectomy due to breast cancer were included in this randomized clinical trial. They were submitted to evaluations in the preoperative period and 07, 15, 30, 60 and 90 days after surgery. They started with limited exercises at $90^{\circ}$ of MA of the shoulder the day after the surgery. Fifteen days later, they were randomized in two groups of 30 patients: Free-Amplitude Group - liberation of joint shoulder amplitude at the threshold of pain, or until feeling the displacement of surgical borders; and Limited Amplitude Group - maintenance of restricted shoulder movements at $90^{\circ}$ until 30 days after the surgery, when they were also cleared for free-amplitude movements. Results: Patients who were cleared for free-amplitude upper limb exercises 15 days after surgery felt less pain, wider shoulder amplitude and better upper limb function, in comparison to those whose movements were restricted to $90^{\circ}$ for 30 days, without increase in incidence and prevalence of postoperative scar complications, and without inference in the need for reoperations. Conclusion: postoperative protocol that clears free MA of shoulder 15 days after surgery is safe and beneficial for the kinetic and functional recovery, as well as algic control in the postoperative period after mastectomy and immediate reconstruction with alloplastic material. 